\title{
Noise Reduction Algorithm Based on Complex Wavelet Transform of Digital Gamma Ray Spectroscopy
}

\author{
Mohamed S. El_Tokhy \\ Electrical Engineering Department, College of Engineering, \\ Aljouf University, \\ Aljouf, KSA \\ engtokhy@gmail.com \\ Imbaby I. Mahmoud \\ Engineering Department, NRC, \\ Atomic Energy Authority, \\ Inshas, Cairo, Egypt
}

\begin{abstract}
This paper investigates the use of complex wavelets in gamma ray spectroscopy signals. In this paper an algorithm for noise elimination of the detected gamma ray spectroscopy signals is studied. This algorithm is based on the complex wavelet transform. Reconstruction of the original detected signal is obtained by applying the inverse complex wavelet transform to the transformed complex wavelet transform signal. Five different cases are studied with different five levels of the complex wavelet transform. Consequently, comparisons between these levels are considered in terms of maximum number of peak heights, execution time, and peak signal to noise ratio (PSNR). Moreover, comparison between different signal reconstruction with respect to different complex wavelet transform levels, size of the transformed signal in each level, and number of coefficient in each subband for certain level. One of the main advantages of this algorithm that discussed in the previous literature is that its filters do not have serious distributed bumps in the wrong side of the power spectrum and, simultaneously, they do not introduce any redundancy to the original signal. The obtained result confirms the high accuracy of the considered algorithm over traditional algorithms for both noise elimination and signal reconstruction.
\end{abstract}

Keywords-Complex Wavelet Transform; Peak Signal-to-Noise Ratio; Linear Filters

\section{INTRODUCTION}

$\mathrm{T}$ HE Scintillation detection experiments indicate that the electronic noise in InI photodetectors was the dominant source of resolution broadening [1]. Hence, the electronic noise behavior of the InI detectors was investigated to determine the magnitude of various noise components in the detectors. These existing noise models are used to analyze the electronic noise in InI detectors. The electronic noise is expected to arise from several sources in InI detectors [1]. These sources are the parallel thermal noise due to the detector leakage current (also commonly referred to as shot noise).

Secondly, the series thermal noise that generated in the channel of the input JFET of the pre-amplifier. Finally, the $1 / f$ noise has been obtained from the detector pre-amplifier assembly. Since the PMT anode signal is very noisy and timing features highly depend on the signal at specific times, a de-noising algorithm is required.
There exist different digital de-noising and smoothing methods (e. g. moving average filters) depending on the application [2]. A de-noising algorithms based on the Wavelet Transform (WT) is implemented to reduce the effect of noise introduced by the noisy analog channel and by the photomultiplier tube as in [2]. In this application, linear smoothing filters are not appropriate because the signal contains a sharp portion associated with the interaction in the first layer (fast component).

Wavelet de-noising which is a non-linear filtering operation analyzes the signal at different time resolution levels and then removes the noise components by thresholding signal components in one or more levels. Depending on the application, the level and threshold should be modified to remove the noise while keeping the important high-frequency components of the signal [2].

Complex wavelet transforms has significant advantages over real wavelet transform for certain signal processing problem [3]. Complex wavelet transforms, in which the real 
and imaginary parts of the transform coefficients are an approximate Hilbert-transform pair, offer three significant advantages over real wavelet transforms: shift invariance, directionality, and explicit phase information. These properties enable efficient statistical models for the coefficients that are also geometrically meaningful [4]. Complex wavelets have not been used widely in signal processing due to the difficulty in designing complex filters which satisfy a perfect reconstruction property [5]. To overcome this Kingsbury [6] proposed a dual-tree implementation of the CWT (DT CWT) which uses two trees of real filters to generate the real and imaginary parts of the wavelet coefficients separately. Even though the outputs of each tree are downsampled by summing the outputs of the two trees during reconstruction, the aliased components of the signal is suppressed and achieved approximate shift invariance [5]. The complex wavelets are first used to perform analysis of the signals [5]. We describe how to extract features to characterize textured signals and test this characterization by resynthesizing textures with matching features. The term de-noising is usually referred to removing the white Gaussian noise or thermal noise which is added to the signal. In our application, this type of noise is mostly introduced by the noisy analog read-out system. In our application, a 5-level de-noising algorithm based on complex wavelet transform functions was used. Also, the rescaling in wavelet decomposition is performed using level-dependent estimation of the noise level. This paper is organized as follows: Section 2 presents the spectroscopy system. The more interesting characteristics of the studied complex wavelet transform are represented in Section 3. Results and discussion are summarized in Section 4 and we terminate our study by a briefly conclusions that we noted from our obtained results.

\section{SYSTEM CONFIGURATION}

In this system, the components of the system for evaluation of noise elimination using complex wavelet transform algorithms are described. Contains the following elements; ${ }^{137} \mathrm{Cs}$ point source, scintillation detector, amplifier, digital system and connection to a desktop personal computer (PC). An 1.5 inches x 7.5 inches NaI(TI) scintillation detector is used to detect the radiation signal from Cs137 point source. This detector is connected to amplifier through coaxial cable which in turn connected to the PC. MATLAB environment is used to perform noise elimination using complex wavelet transform evaluation.

In this paper, an algorithm for noise elimination using complex wavelet transform is studied on digital gamma ray spectroscopy signals. This algorithm is proposed for multidimensional signal processing in [7]. Block diagram showing the algorithm of noise elimination evaluation using the complex wavelet transform is illustrated in Fig. 1. Moreover, different wavelet transform levels are considered.

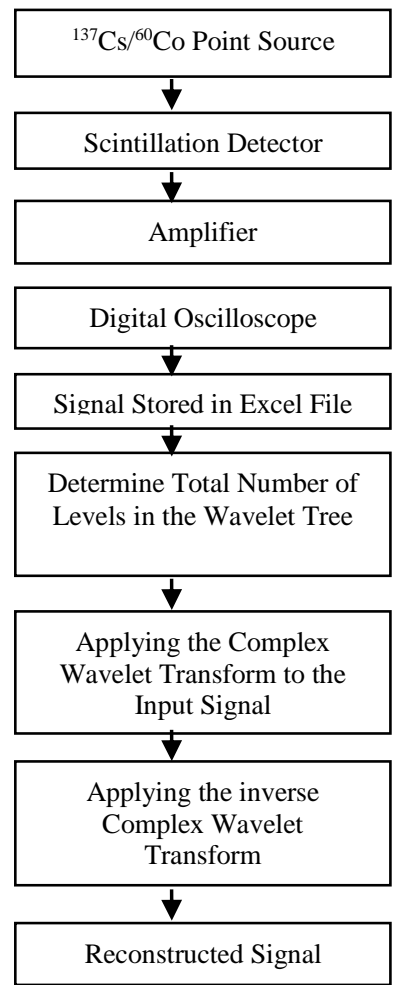

Fig. 1 Block diagram model of the complex wavelet transform algorithm for noise elimination of gamma ray spectroscopy signal

\section{The Complex WAVElet Transform}

\section{A. Linear Phase Filters}

One of the most important properties of the filters which can be applied to 3-band filter banks is linearity of the phase. The phase of the signals which are filtered using the linear phase filters is not perturbed, which means all frequency components of the signal are shifted equally. The filter $\mathrm{h}(\mathrm{t})$, which has the linear phase property, satisfies the following equation[7]

$$
h(n)=\mathrm{e}^{i \theta} h^{*}(N-1-n), 0 \leq n<N-1
$$

where $\mathrm{N}$ and $\theta$ is the length of the filter and an arbitrary variable between zero and $2 \pi$. The following four special kinds of the linear phase filters are used:

1. Real filter with $\theta=0,2 \pi$ and $N$ to be odd,

3. Complex filter with $\theta=\pi / 2,3 \pi / 2$

B.

\section{Filter Bank Characteristics}

Filter banks are widely used in digital signal processing, often integrated in a multirate scheme, to reduce the implementation cost and to improve algorithmic performance [8]. The ideal normalized power spectrum of the filters in the filter bank that satisfies all desirable characteristics, such as having the Hilbert-pairs wavelet 
filters and introducing no bumps on the wrong side of the power spectrum. This filter bank introduces no redundancy because one of the wavelet filters is the complex conjugate of the other, so the resulting coefficients of one filter is the complex conjugate of the other filter, and we can discard them in further analysis [7].

In the ideal case, without considering this low-pass filter, the sampling frequency can be computed using Nyquist's theorem. In the non-ideal case, an anti-aliasing filter must be applied before the sampling part. In this case, the sampling frequency can be calculated using the following equation [7]:

$$
f \geq f_{\mathrm{n}}+2 \mathrm{~B}_{\mathrm{tr}}
$$

where $f_{n}$ is the Nyquist frequency and $B_{\text {tr }}$ is the transient band of the anti-aliasing filter. When using a discrete time low-pass filter after a continuous-to-discrete converter, we can compute another constraint for avoiding distortion. The sampling rate must be computed in such a way that the normalized bandwidth of the analog signal be equal to the pass-band of the low-pass filter. Therefore, the following constraint is computed [7]:

$$
f_{s} \geq \frac{f_{n}}{2 B_{\text {low }}}
$$

where $\mathrm{B}_{\text {low }}$ is the normalized pass-band of the low-pass filter. In this case, the amount of sampling frequency increase with respect to the common sampling is [7]:

$$
R=\frac{f_{n} / 2 B_{\text {low }}}{f_{n}+2 B_{\text {tr }}}
$$

In addition, natural signals decay very fast with frequency increase; therefore if we deviate a little from this sampling rate constraint, the amount of distortion introduces into signal is not very much. We will see this in the second part of the simulation results.

\section{C.}

\section{Filter Bank Design Procedure}

An orthogonal filter bank for digital gamma ray spectroscopy which consists of one real filter $\mathrm{h}_{0}(\mathrm{n})$ and two complex conjugate filters $h_{c}(n)$ and $h_{c}^{*}(n)$ is studied. It is considered [7]

$$
h_{c}(n)=\sqrt{\frac{1}{2}} \times\left(h_{1}(n)+i h_{2}(n)\right)
$$

then for having a complete orthogonal transform, $h_{0}(n)$, $h_{1}(n)$ and $h_{2}(n)$ must satisfy the shift orthogonal condition expressed in the next equation.

In order to have a complete orthogonal transform, the scaling and the wavelet functions must satisfy the shift orthogonal condition.

The design cost function is given by [7]

$$
\Phi=\left(\begin{array}{c}
\sum_{m=0}^{2} \sum_{l} \sum_{i=0}^{2}\left\{h_{i}^{T} Q_{t} h_{m}-\delta(l) \delta(m-i)\right\}^{2}+ \\
\alpha\left\{\begin{array}{l}
h_{0}^{T} M_{0}(\omega) h_{0}+h_{1}^{T} M_{r e}\left(\omega_{2}\right) h_{1}+ \\
h_{2}^{T} M_{r e}\left(\omega_{2}\right) h_{2}+2 h_{2}^{T} M_{i m}\left(\omega_{2}\right) h_{1}
\end{array}\right\}
\end{array}\right)
$$

where $\alpha$ is the weighting coefficient and the stopband power is calculated at those frequencies $\omega 1$ and $\omega 2$ that maximize it. For this purpose, the stopband is sampled uniformly and the stopband power is evaluated at these sampled frequencies. A gradient descend algorithm for minimizing the cost function is used. At every iteration the weighting coefficient $\alpha$ should decrease such that the optimized filters satisfy the shift orthogonal condition. This iteration will terminate when the cost of the shift orthogonal condition expressed in the first line of Equation 6 becomes sufficiently small $\left(\approx 10^{-6}\right)$.

\section{RESUlTS AND DISCUSSION}

We apply the low-pass filter before the analysis and after the synthesis filter banks, therefore we deviate from the perfect reconstruction condition. To observe the amount of distortion introduced into the signal, we apply different levels of the filter bank with the low-pass filter to the acquired signal. The original detected signal is depicted in Fig. 2.

The impulse response of the filters at levels one, two, three, four, and five at different shifts and the resulting absolute value of the wavelet coefficients at these levels are shown in Figs. 3-7, respectively. As illustrated in these figures, these filters are highly oriented in 00, $\pm 450,900$, and 1350 and the real and complex parts of the complex filter constitute Gabor-like filters as in [7]. As illustrated, the filters even at the first level are oriented. It is interesting to test the shift-invariance performance. It is obvious that the studied filter bank has an excellent shift-invariance property which is comparable to the dual-tree complex wavelet transform. For the studied filter bank with the filters of length 37, $\mathrm{Ra}$ is calculated and is shown in Table 3. As an illustrative example, we construct the signal of one, two, three, four, and five levels and the reconstructed signals are shown in Figs. 6-12. One can see that transformed signals are shift-invariant and free of aliasing.

Comparison between the different wavelet levels are depicted in Table 1. From this table, the number of counted peaks decreases with the wavelet level. Also, the number of counted peaks of the original signal is equal to the number counts of level five complex wavelet transform. However, the execution time increases with the level. Also, comparison between different five levels is illustrated in terms of PSNR. From the theoretical results, the PSNR of the reconstructed signals are very high. Therefore, the 
underlined filter bank is considered as a nearly perfect reconstruction filter bank on natural signals. Moreover, comparison between different signal reconstruction in terms of different complex wavelet transform levels, size of the input signal, size of the transformed signal in each level, number of coefficient in each subband for certain level, and total length of the input signal is depicted in Table 2. Also, the normalized amplitude of level 1 complex wavelet transform is depicted in Fig. 13.

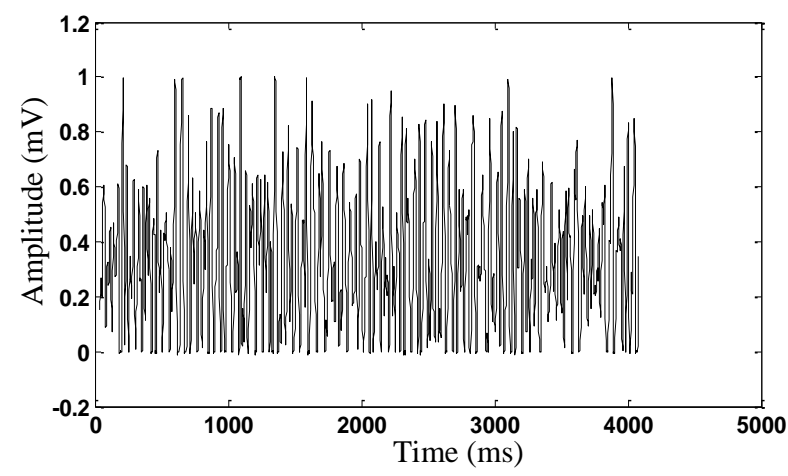

Fig. 2 Original detected signal from the scintillation detector
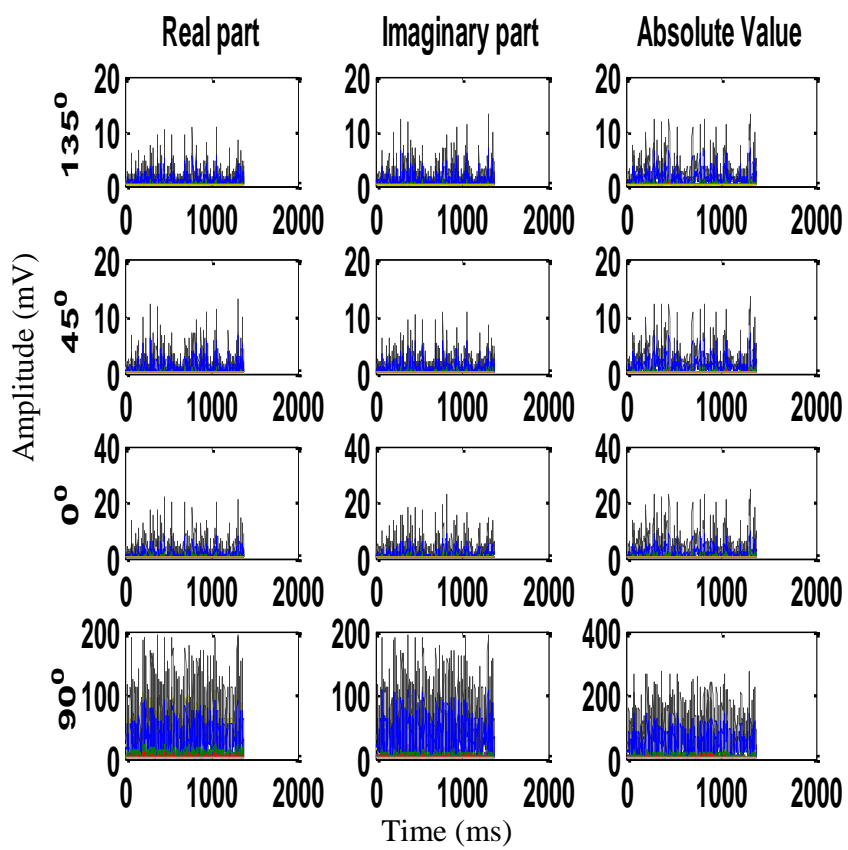

Fig. 3 The impulse response of the filters at level one at different shifts and the resulting absolute value of the wavelet coefficients

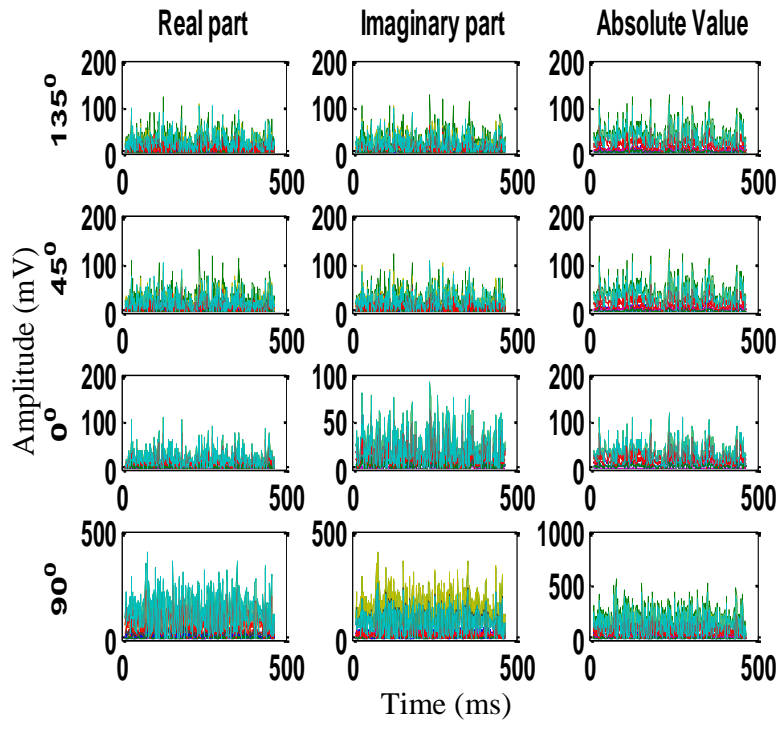

Fig. 4 The impulse response of the filters at level two at different shifts and the resulting absolute value of the wavelet coefficients

TABLE I

COMPARISON BETWEEN DIFFERENT COMPLEX WAVELET TRANSFORM LEVELS

\begin{tabular}{lcc}
\hline \hline & Number of Peaks & $\begin{array}{c}\text { Execution Time } \\
\text { (s) }\end{array}$ \\
\hline Level 1 & 207 & 4.015 \\
Level 2 & 188 & 4.516 \\
Level 3 & 172 & 4.829 \\
Level 4 & 147 & 5.266 \\
Level 5 & 143 & 9.25
\end{tabular}

TABLE 2

COMPARISON BETWEEN DIFFERENT LEVELS IN TERMS OF DIFFERENT STRUCTURE

\begin{tabular}{cccc} 
& \multicolumn{3}{c}{ PARAMETERS } \\
\hline \hline Subbands & $\begin{array}{c}\text { Coefficient } \\
\text { Number }\end{array}$ & Total length \\
\hline Level 1 & {$[4082$ 1] } & 17849 & 160641 \\
Level 2 & {$[2 \times 2$ double $]$} & {$[178495115]$} & 188827 \\
Level 3 & {$[3 \times 2$ double $]$} & {$[178495115$} & 199750 \\
& & $1782]$ & 204007 \\
Level 4 & {$[4 \times 2$ double $]$} & {$[178495115$} & 206009 \\
& & $1782671]$ &
\end{tabular}



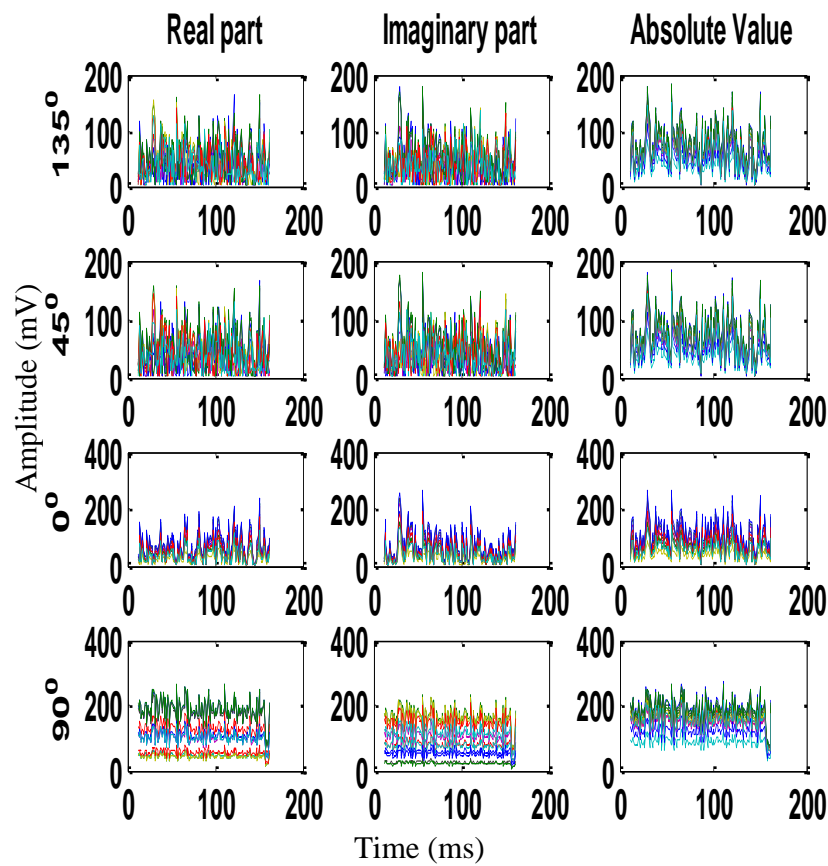

Fig. 5 The impulse response of the filters at level three at different shifts and the resulting absolute value of the wavelet coefficients

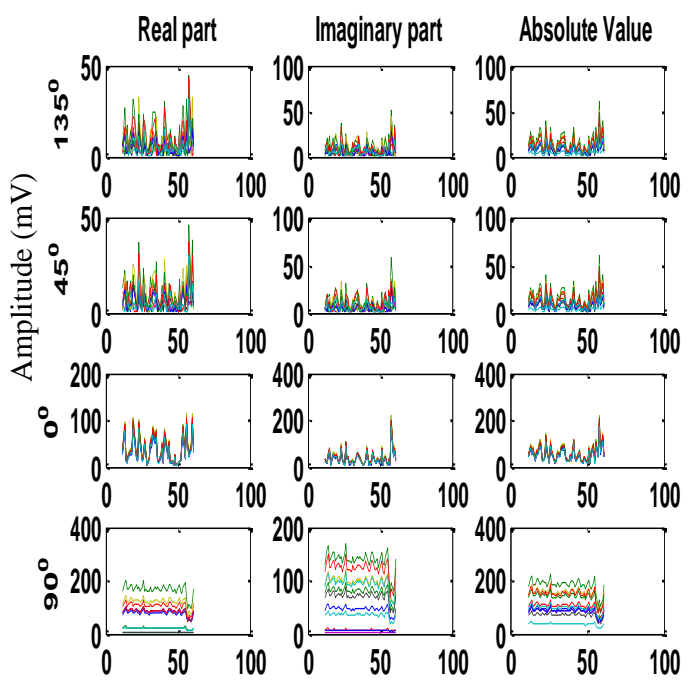

Time (ms)

Fig. 6 The impulse response of the filters at level four at different shifts and the resulting absolute value of the wavelet coefficients

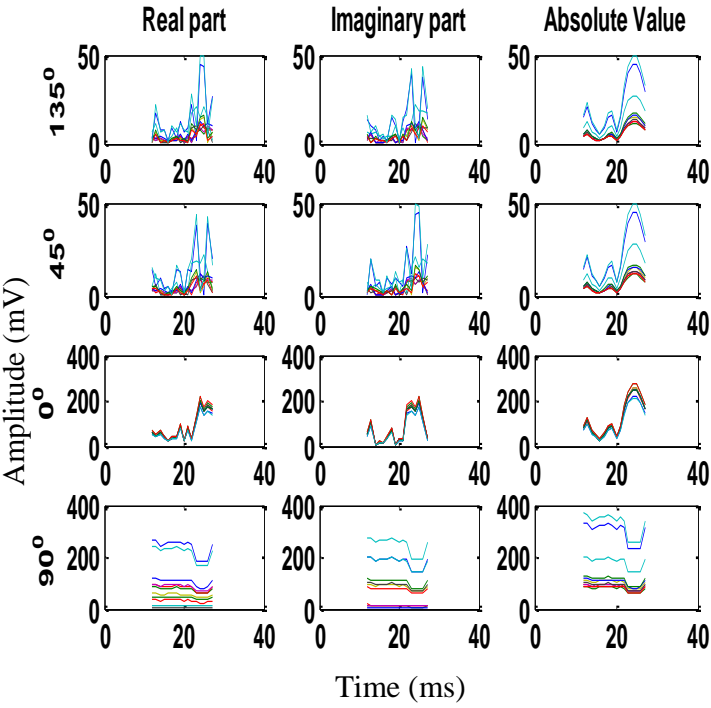

Fig. 7 The impulse response of the filters at level five at different shifts and the resulting absolute value of the wavelet coefficients

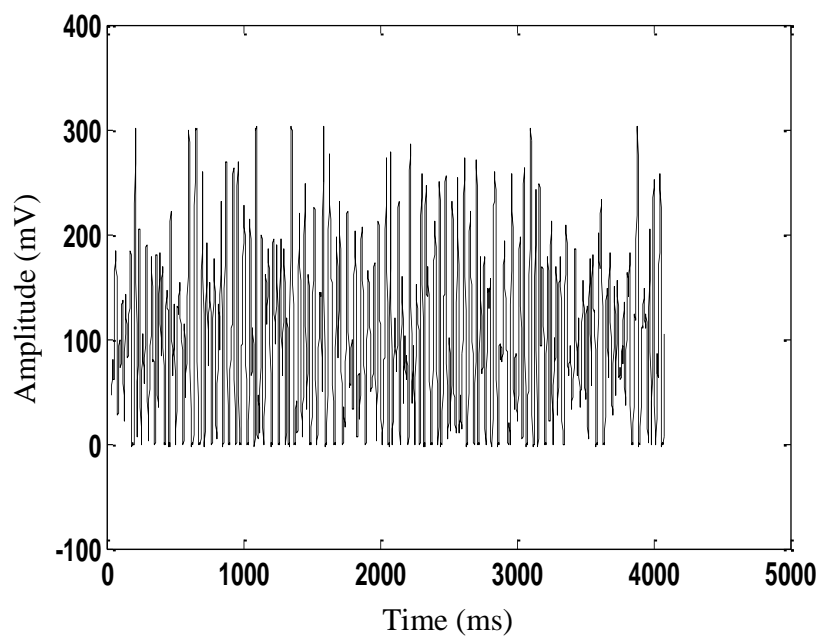

Fig. 8 Reconstructed signal using first level of the algorithm.

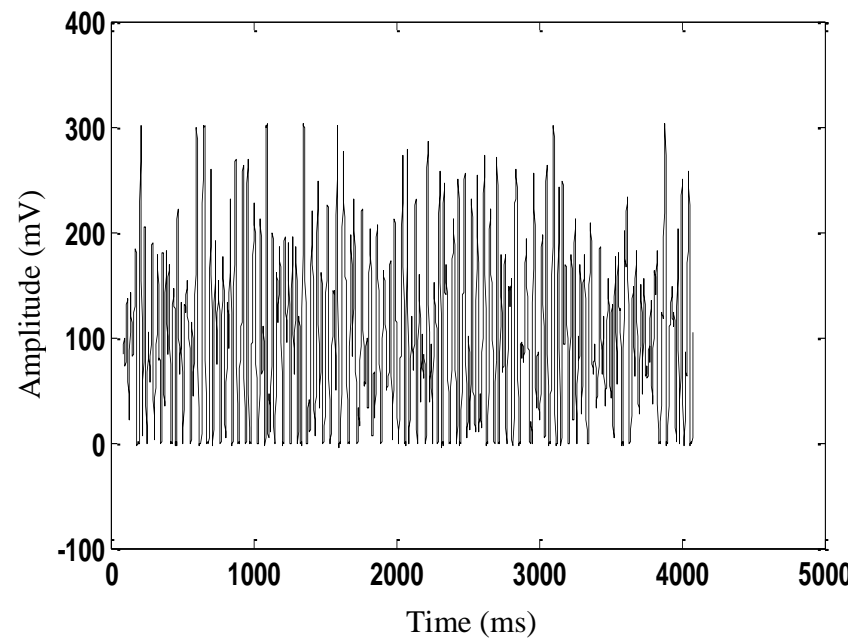


Fig. 9 Reconstructed signal using second levels of the algorithm.

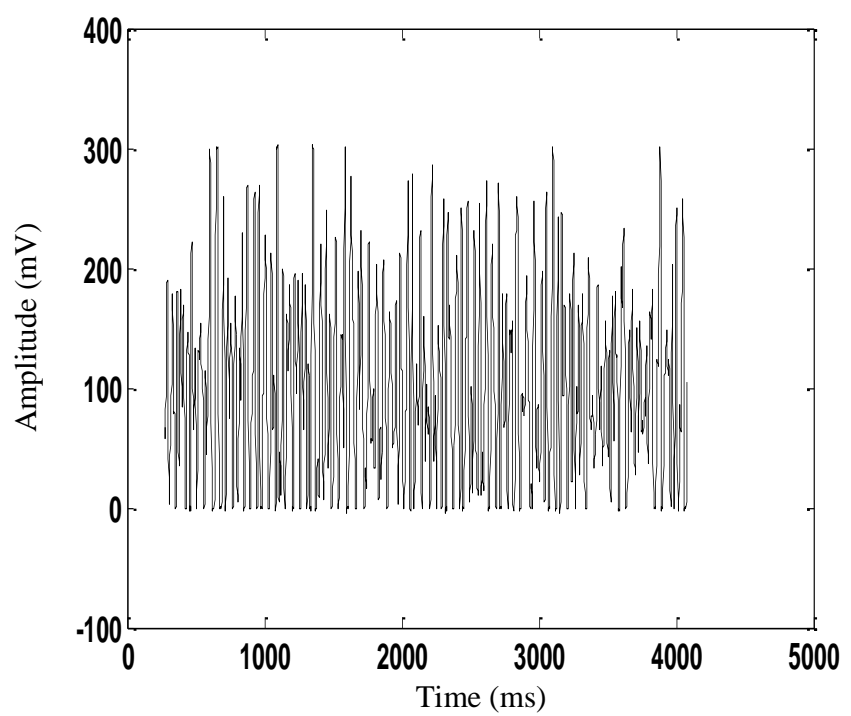

Fig. 10 Reconstructed signal using three levels of the algorithm.

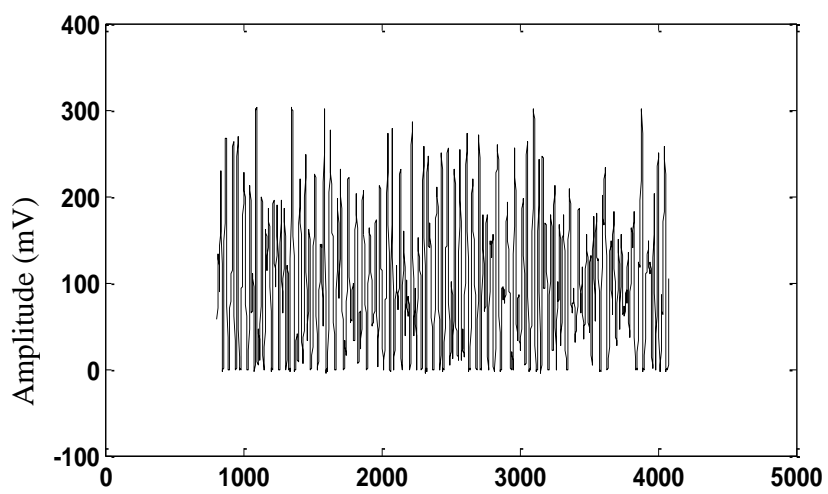

Fig. 11 Reconstructed signal using four levels of the algorithm.

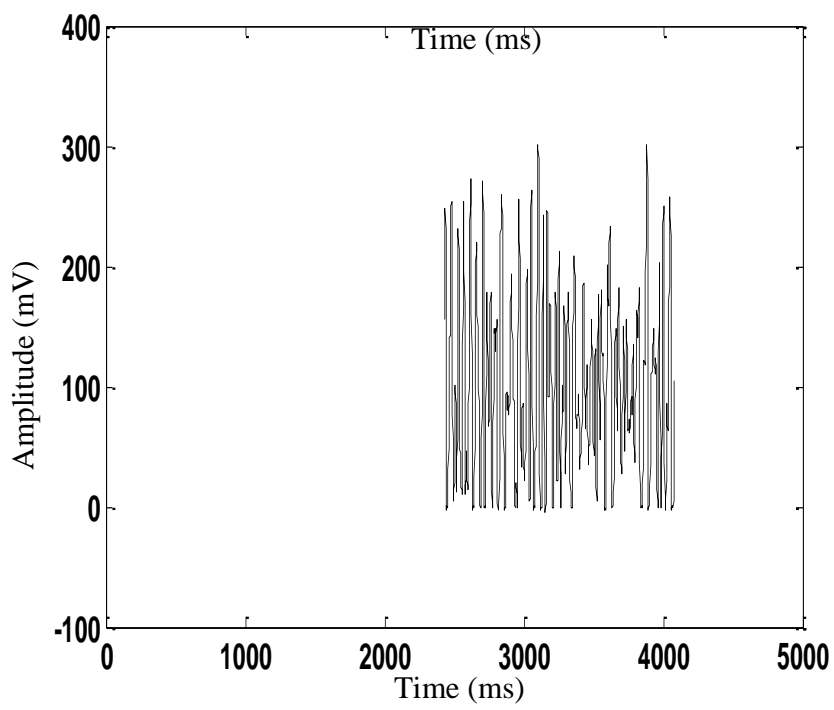

Fig. 12 Reconstructed signal using five levels of the algorithm.

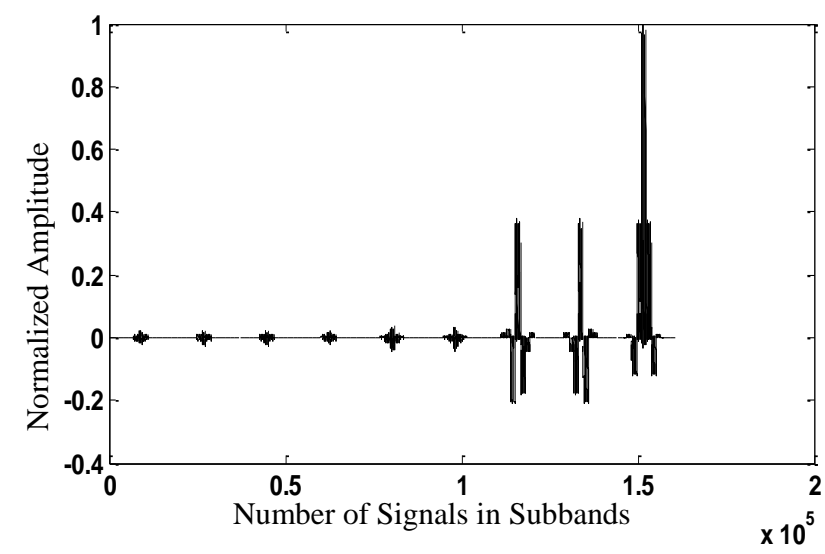

Fig. 13 Normalized amplitude of the output signal.

\section{CONCLUSION}

This paper focuses on noise elimination due to both electronic system readout and coaxial cables between scintillation detector and amplifier. An algorithm based on complex wavelet transforms is studied to do this function. The input signal is transformed using the complex wavelet transform. Then, the inverse complex wavelet transform is applied to the transformed signal. Comparison between different complex wavelet transform levels is considered. This comparison is based on both number of counted peaks, execution time and PSNR. This filter bank has linear phase filters. The resulting filters at different levels do not produce serious bumps on the wrong side of the frequency axis.

\section{REFERENCES}

[1] K. S. Shah, P. Bennett, L. P. Moy, M. M. Misra, W. W. Moses, "Characterization of Indium Iodide Detectors for scintillation studies", Nuclear Instruments and Methods in Physics Research A, Vol. 380, pp. 215-219, 1996.

[2] Siavash Yousefi and Luca Lucchese, "Digital Pulse Shape Discrimination in Triple-Layer Phoswich Detectors Using Fuzzy Logic", IEEE Transactions on Nuclear Science, Vol. 55, No. 5, October 2008.

[3] H. N. Abdullah, "SAR image denoising based on dual-tree complex wavelet transform", Journal of Engineering and Applied Science, Vol. 3, No. 7, pp. 587-590, 2008.

[4] Felix C. A. Fernandes,t Michael B. Wakinr and Richard G. Baraniukr, "Non-redundant, linear-phase, semi-orthogonal, directional complex wavelets", ICASSP, IEEE, 2004, pp. II - 953- II - 956.

[5] Musoko Victor, Prochazka ales, "Complex wavelet transform in signal and image analysis", $14^{\text {th }}$ International Scientific - Technical conference on Process Control, Czech Republic, June 2004.

[6] N. G. Kingsbury, "Complex wavelets for shift invariant analysis and filtering of signals", Journal of Applied and Computational Harmonic Analysis, Vol 10, No 3, pp. 234-253, 2001.

[7] Reshad Hosseini, Mansur Vafadust, "Almost Perfect Reconstruction Filter Bank for Non-redundant, Approximately Shift-Invariant, Complex Wavelet Transforms", Journal of Wavelet Theory and Applications, Vol. 2, No. 1, pp. 1-14, 2008.

[8] Koen. Eneman, and Marc. Moonen, "DFT modulated filter bank design for oversampled subband systems", Signal Processing Journal, Vol. 81 No.9, pp.1947-1973, 2001. 